\title{
Kinematic Modelling of Wheeled Mobile Manipulators
}

\author{
B. Bayle ${ }^{\star}$, J.-Y. Fourquet ${ }^{\star}$ and M. Renaud ${ }^{\diamond}$ \\ ${ }^{\star}$ LSIIT, Strasbourg, France, ${ }^{\star \star}$ ENIT, Tarbes, France, ${ }^{\diamond}$ LAAS-CNRS, Toulouse, France,
}

\begin{abstract}
We propose a systematic modelling of the nonholonomic mobile manipulators built from a robotic arm mounted on a wheeled mobile platform. It extends the fundamental notions of nonholonomy, mobility and manœuvrability to the case of these hybrid holonomic/nonholonomic systems. It offers unambiguous definitions and models to the designer of kinematic control laws.
\end{abstract}

\section{Introduction}

For an "intelligent" robot, most imaginable tasks require locomotion and manipulation abilities. Mobile manipulators combine these abilities, thus extending workspace for manipulation, allowing new strategies to help navigation, etc. Additionally they seem particularly suitable for human-like tasks. Different categories of mobile manipulators have been designed in the past ten years. They are mainly differentiated by their mode of locomotion [2, 4] and then classified according to their platforms: walking robots, wheeled platforms, sub-sea systems, free-flying space robots, etc.

In this paper we consider the case of wheeled mobile manipulators, which represent an important part of mobile manipulators involved in indoor robotics. These systems are surely the simplest. However, they are subjected to constraints - termed as nonholonomic- due to the rolling without slipping (r.w.s.) of the wheels on the ground. In spite of the early study of nonholonomic platforms [9, 1], the systematic modelling of these systems is quite recent [7]. It may be the reason why all the studies of wheeled mobile manipulators, to our knowledge, deal with a particular system. Most of the time this system is based on a platform with two independently driven wheels. The aim of this paper is to extend the State of the Art in modelling to provide convenient tools to describe the geometry and the kinematic modelling of wheeled mobile manipulators. We first model independently the platform in paragraph 2.1 and then the robotic arm in paragraph 2.2. The modelling of the mobile manipulator will be finally established in part 3. This article aims to be pedagogic. Thus, every notion is illustrated through the example of the planar mobile manipulator represented in Figure 1, built from a car-like platform and a two-joint robotic arm.

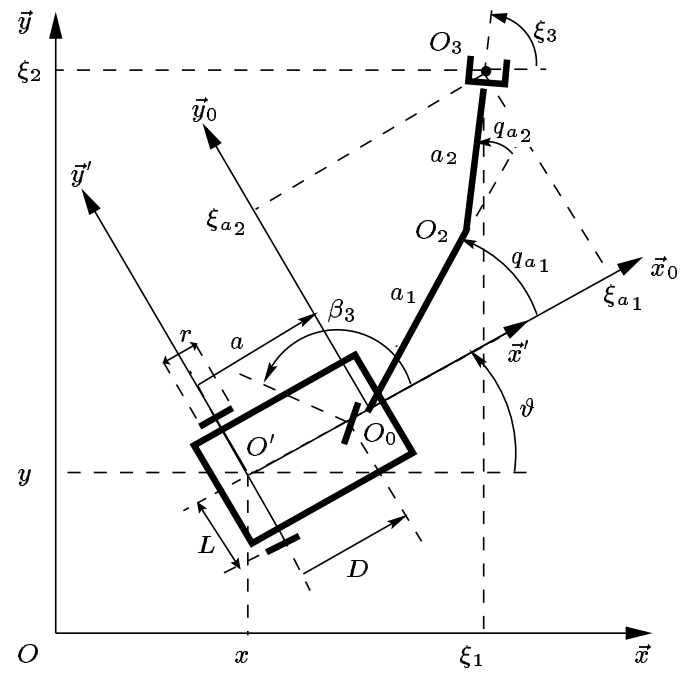

Figure 1: Planar mobile manipulator with a car-like platform

\section{Modelling}

\subsection{Wheeled mobile platforms}

The wheeled mobile platforms are properly described and modelled by G. Campion et al. in [7]. In this section, we introduce only the elements which are necessary in our study. Only minor changes have been done purposely to be consistent with the modelling of mobile manipulators.

\subsubsection{Description of the platform}

We assume that the mobile platform moves on a planar horizontal surface. Let $\mathcal{R}=(O, \vec{x}, \vec{y}, \vec{z})$ be any fixed frame with $\vec{z}$ vertical and $\mathcal{R}^{\prime}=\left(O^{\prime}, \vec{x}^{\prime}, \vec{y}^{\prime}, \vec{z}^{\prime}\right)$ a mobile frame linked to the platform. The origin of $\mathcal{R}^{\prime}$ is usually chosen as a remarkable point of this platform (e.g. the midpoint of the rear axle).

The location ${ }^{1}$ of the platform is given by the $m_{p}=3-$ dimensional vector $\boldsymbol{\xi}_{p}=\left[\begin{array}{lll}x & y & \vartheta\end{array}\right]^{T}$, where $x$ and $y$ are respectively the abscissa and the ordinate of $O^{\prime}$ in $\mathcal{R}$ and $\vartheta$

\footnotetext{
${ }^{1}$ It is termed as posture in [7] but the choice of location allows us to use the same name for the robotic arms, the platforms and later for the mobile manipulators.
} 
the angle $\left(\vec{x}, \vec{x}^{\prime}\right)$. The set of all the locations constitutes the location space of the platform, denoted by $\mathcal{M}_{p}$.

The mobile platforms wheels can be classified in four types (see Figure 2):

- the fixed wheels for which the axle has a fixed direction;

- the steering wheels, for which the orientation axis passes through the center of the wheel;

- the castor wheels, for which the orientation axis does not pass through the center of the wheel;

- the Swedish wheels, which are similar to the fixed wheels, with the exception of an additional parameter $(\gamma)$ that describes the direction, with respect to the wheels plane, of the zero component of the velocity at the contact point [7].

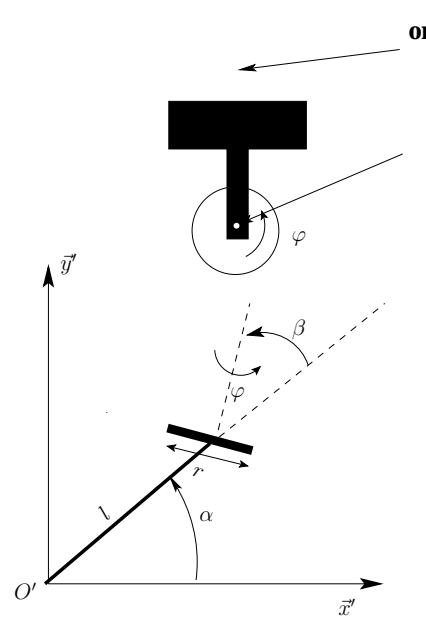

fixed and steering wheels orientation axis axle

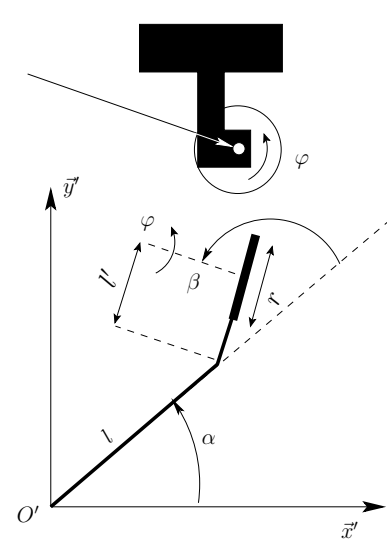

castor wheels
Figure 2: Different types of wheels

It is assumed that the wheels always keep their shape which is sensible in indoor robotics - and that there is always r.w.s. of the wheels on the ground.

Let $N=N_{f}+N_{s}+N_{c}+N_{s w}$ be the total number of wheels. $N_{f}, N_{s}, N_{c}, N_{s w}$ represent respectively the number of fixed, steering, castor and Swedish wheels. From now on, these indexes correspond to the different types of wheels. Let $\varphi_{f}, \boldsymbol{\varphi}_{s}, \boldsymbol{\varphi}_{c}, \boldsymbol{\varphi}_{s w}$ be the vectors giving the rotation angles of the wheels about their horizontal axle: all of them are variables. Let $\boldsymbol{\beta}_{f}, \boldsymbol{\beta}_{s}, \boldsymbol{\beta}_{c}$ and $\boldsymbol{\beta}_{s w}$ be the vectors giving the orientation of the wheels: $\boldsymbol{\beta}_{f}$ and $\boldsymbol{\beta}_{s w}$ are constants whereas $\boldsymbol{\beta}_{s}$ and $\boldsymbol{\beta}_{c}$ are variables. The

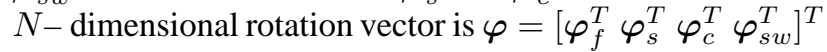
The platform configuration is known when the position of all its points in $\mathcal{R}$ are known [10]. It is given by the $n_{p}$-dimensional vector $\boldsymbol{q}_{p}=\left[\boldsymbol{\varphi}^{T} \boldsymbol{\xi}_{p}^{T} \boldsymbol{\beta}_{s}^{T} \boldsymbol{\beta}_{c}^{T}\right]^{T}$, with $n_{p}=N+m_{p}+N_{s}+N_{c}$. The set of all the configurations constitutes the configuration space of the platform, denoted by $\mathcal{N}_{p}$.
The platforms are then described by the type of the different wheels, and by their geometry: the angles $\alpha, \beta, \gamma$ and the lengths $l, l^{\prime}$ and $r$.

\subsubsection{Rolling without slipping constraints}

Let $R(\vartheta)$ be the 3 -order rotation matrix expressing the orientation of $\mathcal{R}^{\prime}$ with respect to $\mathcal{R}$.

According to Figure 2 the r.w.s. constraints can always be written:

$$
\begin{array}{r}
J_{1}\left(\boldsymbol{\beta}_{s}, \boldsymbol{\beta}_{c}\right) R^{T}(\vartheta) \dot{\boldsymbol{\xi}}_{p}+J_{2} \dot{\boldsymbol{\varphi}}=\mathbf{0}, \\
C_{1}\left(\boldsymbol{\beta}_{s}, \boldsymbol{\beta}_{c}\right) R^{T}(\vartheta) \dot{\boldsymbol{\xi}}_{p}+C_{2} \dot{\boldsymbol{\beta}}_{c}=\mathbf{0},
\end{array}
$$

where the associated matrices are given in [7].

Equation (1) expresses the constraint in the vertical plane of the wheel and equation (2) in the vertical plane orthogonal to the wheel; they are said to be nonholonomic since they cannot be integrated.

\subsubsection{Instantaneous kinematic models}

If we take into account the structures of $C_{1}$ and $C_{2}$ we can separate (2) in two parts:

$$
\begin{array}{r}
C_{1}^{\star}\left(\boldsymbol{\beta}_{s}\right) R^{T}(\vartheta) \dot{\boldsymbol{\xi}}_{p}=\mathbf{0} \\
C_{1 c}\left(\boldsymbol{\beta}_{c}\right) R^{T}(\vartheta) \dot{\boldsymbol{\xi}}_{p}+C_{2 c} \dot{\boldsymbol{\beta}}_{c}=\mathbf{0} .
\end{array}
$$

Equation (3) is such that $R^{T}(\vartheta) \dot{\boldsymbol{\xi}}_{p}$ must be an element of $C_{1}^{\star}\left(\boldsymbol{\beta}_{s}\right)$ null space, denoted by $\operatorname{Ker}\left(C_{1}^{\star}\left(\beta_{s}\right)\right)$. Let $\Sigma\left(\boldsymbol{\beta}_{s}\right)$ be a matrix the columns of which are a basis of this $\delta_{m_{p}}$ - dimensional null space, with: $\delta_{m_{p}}=\operatorname{dim}\left(\operatorname{Ker}\left(C_{1}^{\star}\left(\beta_{s}\right)\right)=3-\operatorname{rank} C_{1}^{\star}\left(\beta_{s}\right)\right.$. $\delta_{m_{p}}$ is termed as the degree of mobility of the mobile platform [7]. From the previous calculus, we conclude that there exists a $\delta_{m_{p}}-$ dimensional vector $\boldsymbol{\eta}_{p}$ such that:

$$
\dot{\boldsymbol{\xi}}_{p}=R(\vartheta) \Sigma\left(\boldsymbol{\beta}_{s}\right) \boldsymbol{\eta}_{p}
$$

We will term it as the control of mobility of the platform and equation (5) forms the instantaneous location kinematic model (ILKM) of the mobile platform. As $C_{1}^{\star}\left(\boldsymbol{\beta}_{s}\right)=\left[C_{1 f}^{T} C_{1 s}^{T}\left(\boldsymbol{\beta}_{s}\right)\right]^{T}$ [7], we write (3) as:

$$
C_{1 f} R^{T}(\vartheta) \dot{\boldsymbol{\xi}}_{p}=\mathbf{0} \text { and } C_{1 s}\left(\boldsymbol{\beta}_{s}\right) R^{T}(\vartheta) \dot{\boldsymbol{\xi}}_{p}=\mathbf{0} .
$$

$\delta_{s_{p}}=\operatorname{rank} C_{1 s}\left(\boldsymbol{\beta}_{s}\right)$ is the degree of steerability of the mobile platform and $\delta_{M_{p}}=\delta_{m_{p}}+\delta_{s_{p}}=\delta_{m_{p}}+N_{s}$ is the degree of manoeuvrability of the mobile platform [7] (We assume that we control the velocity of the steering wheels around their orientation axis. Thus, it is necessary to have $N_{s}=\delta_{s_{p}}$ to control independently the $N_{s}$ steering wheels). As a consequence the $\delta_{s_{p}}$-dimensional vector $\zeta_{p}$ is termed as the control of steerability of the mobile platform:

$$
\dot{\boldsymbol{\beta}}_{s}=\boldsymbol{\zeta}_{p},
$$


Now, when $\left(\dot{\boldsymbol{\xi}}_{p}, \dot{\boldsymbol{\beta}}_{s}\right)$ ( or equivalently $\left(\boldsymbol{\eta}_{p}, \boldsymbol{\zeta}_{p}\right)$ with (5) and (6)) are known, the whole generalized velocity $\dot{\boldsymbol{q}}_{p}$ is known from (1) (2). If we define the control of manoeuvrability of the mobile platform by the $\delta_{M_{p}}$-dimensional vector $\boldsymbol{u}_{p}=\left[\begin{array}{ll}\boldsymbol{\eta}_{p}^{T} & \boldsymbol{\zeta}_{p}^{T}\end{array}\right]^{T}$, the instantaneous configuration kinematic model (ICKM) of the platform [7] writes:

$$
\dot{\boldsymbol{q}}_{p}=S_{p}\left(\vartheta, \boldsymbol{\beta}_{s}, \boldsymbol{\beta}_{c}\right) \boldsymbol{u}_{p}
$$

From now on, we assume that the mobile platform always has a number of actuators equal to its degree of manoeuvrability.

Example. We consider the car-like platform of Figure 1. This vehicle is equivalent to a car with only one steering wheel in the front, on the longitudinal axis of the vehicle ${ }^{2}$, and two fixed wheels on the same axle, in the back. The wheels are numbered as follows: $1=$ left back wheel, $2=$ right back wheel, $3=$ front wheel. We denote by $\beta_{3}=\beta_{s_{1}}$ the orientation of the steering wheel, by $\varphi_{1}=\varphi_{f_{1}}, \varphi_{2}=$ $\varphi_{f_{2}}$ and $\varphi_{3}=\varphi_{s_{1}}$ the rotation angles of the right fixed wheel, of the left fixed wheel and of the steering wheel (we hide indexes.$_{f}$ and.$_{s}$ in order to simplify writings). So we

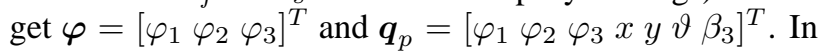
the following, we write: $C_{\vartheta}=\cos \vartheta, S_{\vartheta}=\sin \vartheta, C_{\beta_{3}}=$ $\cos \beta_{3}$ and $S_{\beta_{3}}=\sin \beta_{3}$.

In this case, equations (1) and (2) write:

$$
\begin{array}{r}
J_{1}\left(\beta_{3}\right) R^{T}(\vartheta) \dot{\boldsymbol{\xi}}_{p}+J_{2} \dot{\boldsymbol{\varphi}}=\mathbf{0}, \\
C_{1}\left(\beta_{3}\right) R^{T}(\vartheta) \dot{\boldsymbol{\xi}}_{p}=\mathbf{0},
\end{array}
$$

with (see Figure 1):

$$
\begin{array}{r}
J_{1}\left(\beta_{3}\right)=\left[\begin{array}{ccc}
1 & 0 & L \\
-1 & 0 & L \\
-S_{\beta_{3}} & C_{\beta_{3}} & D C_{\beta_{3}}
\end{array}\right], J_{2}=\left[\begin{array}{ccc}
r & 0 & 0 \\
0 & r & 0 \\
0 & 0 & r
\end{array}\right] \\
\text { and } C_{1}^{\star}\left(\beta_{3}\right)=\left[\begin{array}{ccc}
0 & -1 & 0 \\
0 & 1 & 0 \\
C_{\beta_{3}} & S_{\beta_{3}} & D S_{\beta_{3}}
\end{array}\right]
\end{array}
$$

From (11), we know that $\operatorname{Ker}\left(C_{1}^{\star}\left(\beta_{3}\right)\right)$ is 1 - dimensional. A base of this null space is given by $\left[-D S_{\beta_{3}} 0 C_{\beta_{3}}\right]^{T}$ and then:

$$
R^{T}(\vartheta) \dot{\boldsymbol{\xi}}_{p}=\left[\begin{array}{lll}
-D S_{\beta_{3}} & 0 & C_{\beta_{3}}
\end{array}\right]^{T} \eta_{p},
$$

where $\eta_{p}$ is a scalar, then $\delta_{m_{p}}=1$. Finally:

$$
\dot{\boldsymbol{\xi}}_{p}=\left[\begin{array}{lll}
-D C_{\vartheta} S_{\beta_{3}} & -D S_{\vartheta} S_{\beta_{3}} & C_{\beta_{3}}
\end{array}\right]^{T} \eta_{p} .
$$

It is the ILKM of the mobile platform. We notice that the derivative of the platform location depends on the steering

\footnotetext{
${ }^{2}$ This model is convenient to describe the system equipped with two front wheels sharing the same instantaneous center of rotation.
}

wheel orientation $\beta_{3}$, the derivative of which can be chosen independently as $\dot{\beta}_{3}=\zeta_{p}$, then $\delta_{s_{p}}=1$. In this case, $\boldsymbol{u}_{p}=\left[\eta_{p} \zeta_{p}\right]^{T}$ and $\delta_{M_{p}}=2$.

The platform ICKM computation is detailed in [3]:

$$
\dot{\boldsymbol{q}}_{p}=\left[\begin{array}{lll}
\dot{\boldsymbol{\varphi}} & \dot{\boldsymbol{\xi}}_{p} & \dot{\beta}_{3}
\end{array}\right]^{T}=S_{p}\left(\vartheta, \beta_{3}\right) \boldsymbol{u}_{p}
$$

with: $S_{p}\left(\vartheta, \beta_{3}\right)=\left[\begin{array}{cc}\frac{1}{r}\left(D S_{\beta_{3}}-L C_{\beta_{3}}\right) & 0 \\ -\frac{1}{r}\left(D S_{\beta_{3}}+L C_{\beta_{3}}\right) & 0 \\ -\frac{D}{r} & 0 \\ -D C_{\vartheta} S_{\beta_{3}} & 0 \\ -D S_{\vartheta} S_{\beta_{3}} & 0 \\ C_{\beta_{3}} & 0 \\ 0 & 1\end{array}\right]$.

\subsection{Robotic arms}

\subsubsection{Description of the robotic arm}

We consider a robotic arm built from $n_{a}$ mobile bodies (supposed perfectly rigid) articulated by $n_{a}$ rotoïd and/or prismatic joints. The most usual way to model a robotic arm consists in using the Denavit-Hartenberg modified parameters [8]. We associate the frame $\mathcal{R}_{i}=\left(O_{i}, \vec{x}_{i}, \vec{y}_{i}, \vec{z}_{i}\right)$, with $i=0,1, \ldots, n_{a}$, to the $i$-th body of the robotic arm. So, the frame $\mathcal{R}_{0}$ is linked to the base. The center of the EE (tool or grip) is denoted by $O_{n_{a}+1}$. So, both points $O_{n_{a}}$ and $O_{n_{a}+1}$ are linked to the EE (see Figure 3).

The robotic arm configuration is known when the position of all its points in $\mathcal{R}_{0}$ are known. It is given by the $n_{a}$-dimensional vector $\boldsymbol{q}_{a}=\left[\begin{array}{llll}q_{a_{1}} & q_{a_{2}} & \ldots & q_{a_{n_{a}}}\end{array}\right]^{T}$ which characterize the values of the different joints: rotation angles for the rotoïd ones, translations for the prismatic ones. The set of all the configurations constitutes the configuration space of the robotic arm, denoted by $\mathcal{N}_{a}$.

The location of the robotic arm $\mathrm{EE}$ is given by a $m_{a}$-dimensional vector $\boldsymbol{\xi}_{a}=\left[\begin{array}{llll}\xi_{a_{1}} & \xi_{a_{2}} & \ldots & \xi_{a_{m_{a}}}\end{array}\right]^{T}$, where $\xi_{a_{1}}, \xi_{a_{2}}, \ldots, \xi_{a_{m_{a}}}$ define the position and the orientation of the EE in $\mathcal{R}_{0}$. The set of all the locations constitutes the location space of the robotic arm EE, denoted by $\mathcal{M}_{a}$. This location is defined according to the task to achieve. So, for a planar problem, we will consider only the EE position and orientation in the plane. In the most general case we chose the Cartesian coordinates of the point $O_{n_{a}+1}$ in $\mathcal{R}_{0}$ for $\xi_{a_{1}}, \xi_{a_{2}}$ and $\xi_{a_{3}}$ and the classical Euler angles [11] for $\xi_{a_{4}}=\psi, \xi_{a_{5}}=\theta$ and $\xi_{a_{6}}=\varphi$.

\subsubsection{Models of a robotic arm}

The kinematic model (KM) sets the location of its EE as a function of its configuration:

$$
\begin{aligned}
\boldsymbol{f}_{a}: \quad \mathcal{N}_{a} & \longrightarrow \mathcal{M}_{a} \\
\boldsymbol{q}_{a} & \longmapsto \boldsymbol{\xi}_{a}=\boldsymbol{f}_{a}\left(\boldsymbol{q}_{a}\right),
\end{aligned}
$$

and the instantaneous kinematic model (IKM) the derivative of its location as a function of the derivative of its con- 
figuration:

$$
\begin{aligned}
J_{a}\left(\boldsymbol{q}_{a}\right): T_{\boldsymbol{q}_{a}} \mathcal{N}_{a} & \longrightarrow T_{\boldsymbol{\xi}_{a}} \mathcal{M}_{a} \\
\dot{\boldsymbol{q}}_{a} & \longmapsto \dot{\boldsymbol{\xi}}_{a}=J_{a}\left(\boldsymbol{q}_{a}\right) \dot{\boldsymbol{q}}_{a} .
\end{aligned}
$$

This last model uses the Jacobian matrix $J_{a}\left(\boldsymbol{q}_{a}\right)$ of the function $\boldsymbol{f}_{a}: J_{a}\left(\boldsymbol{q}_{a}\right)=\frac{\partial \boldsymbol{f}_{a}}{\partial \boldsymbol{q}_{a}}$. The configurations such that the rank of $J_{a}\left(\boldsymbol{q}_{a}\right)$ decreases are singular kinematic configurations and the problem, robotic arm and task, is redundant when $n_{a}>m_{a}$.

We define the vector of the kinematic control of the robotic arm by:

$$
\boldsymbol{u}_{a}=\dot{\boldsymbol{q}}_{a} .
$$

\section{Modelling of mobile manipulators}

In the case of holonomic manipulators, IKM are used. They are linear maps - for a given configuration - between the derivatives of location and configuration. These models are used for velocity control schemes and motion generation. The associated notions of redundancy, manipulability [12] and singular configurations allow to answer locally the following questions:

- Redundancy: is there a choice in the motion of the mechanical system for a given motion of the EE ? And, what is the dimension of the space of solutions?

- Singular configurations: for which configurations - when they exist - does the dimension of the available EE velocities decrease?

- Manipulability: for a given configuration, what is the ability of the system to provide EE velocities in any directions?

In the case of wheeled mobile manipulators, we are led to define IKM and to reconsider the previous notions. Nonetheless, it can be remarked that concerning wheeled systems, the different components of the configuration play a different role. Indeed:

- Generally, we are not interested in imposing the angular values $\varphi$ of the wheels but rather in fixing the location of the platform. Moreover, the ability to produce EE velocities does not depend on this value. In many cases, two platform configurations for which only the angles $\varphi$ differ share the same properties and are not distinguishable from the task viewpoint.

- When the system has steering wheels, the ability to produce EE velocities depends on the orientation value $\boldsymbol{\beta}_{s}$ but not on its derivative. Moreover, when no dynamic phenomena are taken into account, the steerability control is independent of any component of the configuration. Thus we are led to consider IKM in location and configuration and to characterize a kinematic control vector made of two parts: a mobility control vector - the only one that acts on the EE velocities - and a manoeuvrability control vector taking into account the steering velocity of the wheels when it exists.

\subsection{Description of the mobile manipulator}

We consider the general case of a mobile manipulator built from a mobile platform equipped with an on-board robotic arm, introduced respectively in paragraph 2.1 and 2.2. The coordinates of $O_{0}$ in $\mathcal{R}^{\prime}$ are given by $\left[\begin{array}{lll}a & b & 0\end{array}\right]^{T}$ (see Figure 3).
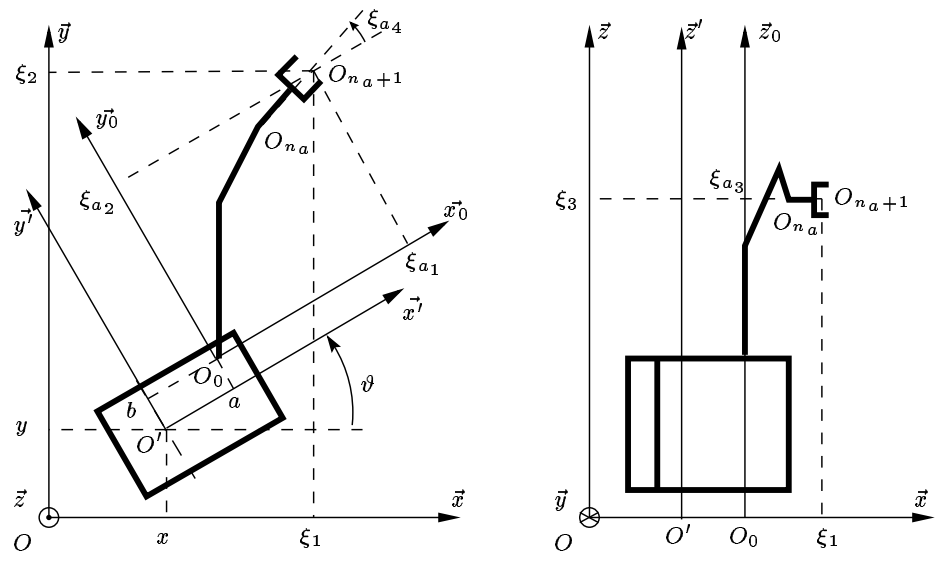

Figure 3: Geometry of mobile manipulators

The mobile manipulator configuration is known when the position of all its points in $\mathcal{R}$ are known. It is defined by the $n$-dimensionnal vector $\boldsymbol{q}=\left[\begin{array}{llll}q_{1} & q_{2} & \ldots & q_{n}\end{array}\right]^{T}=\left[\begin{array}{ll}\boldsymbol{q}_{a}^{T} & \boldsymbol{q}_{p}^{T}\end{array}\right]^{T}$ with $n=n_{a}+n_{p}$. The set of all the configurations constitutes the configuration space of the mobile manipulator, denoted by $\mathcal{N}$.

The location of the mobile manipulator EE is given by a $m$-dimensional vector $\boldsymbol{\xi}=\left[\begin{array}{llll}\xi_{1} & \xi_{2} & \ldots & \xi_{m}\end{array}\right]^{T}$, where $\xi_{1}$, $\xi_{2}, \ldots, \xi_{m}$ define the position and the orientation of the EE in $\mathcal{R}$. The set of all the locations constitutes the location space of the mobile manipulator $\mathrm{EE}$, denoted by $\mathcal{M}$. This location is defined according to the task to achieve. So, for a planar problem, we will consider only the EE position and orientation in the plane. In the most general case we choose the Cartesian coordinates of the point $O_{n_{a}+1}$ in $\mathcal{R}$ for $\xi_{1}, \xi_{2}$ and $\xi_{3}$ and the classical Euler angles for $\xi_{4}=\beta$, $\xi_{5}=\theta$ and $\xi_{6}=\varphi$. Remark that $\beta=\vartheta+\psi$; this property justifies the choice of the Euler classical angles.

\subsection{Models of a mobile manipulator}

The kinematic model (KM) sets the location of its EE as a function of the robotic arm configuration and of the platform location:

$$
\begin{aligned}
\boldsymbol{f}: \mathcal{N}_{a} \times \mathcal{M}_{p} & \longrightarrow \mathcal{M} \\
\left(\boldsymbol{q}_{a}, \boldsymbol{\xi}_{p}\right) & \longmapsto \boldsymbol{\xi}=\boldsymbol{f}\left(\boldsymbol{q}_{a}, \boldsymbol{\xi}_{p}\right) .
\end{aligned}
$$

The shape of the mobile manipulators we are studying is shown in Figure 3, regardless of the type of wheels. For any wheeled mobile platform with location $\boldsymbol{\xi}_{p}=\left[\begin{array}{lll}x & y & \vartheta\end{array}\right]^{T}$ - equipped with a robotic arm with configuration $\boldsymbol{q}_{a}$ and 
location $\boldsymbol{\xi}_{a}$ - the position of the mobile manipulator EE in $\mathcal{R}$ is:

$$
\begin{aligned}
& \xi_{1}=x+\left(a+\xi_{a_{1}}\right) C_{\vartheta}-\left(b+\xi_{a_{2}}\right) S_{\vartheta}, \\
& \xi_{2}=y+\left(a+\xi_{a_{1}}\right) S_{\vartheta}+\left(b+\xi_{a_{2}}\right) C_{\vartheta}, \\
& \xi_{3}=\xi_{a_{3}}
\end{aligned}
$$

and its orientation:

$$
\begin{aligned}
& \xi_{4}=\beta=\vartheta+\psi, \\
& \xi_{5}=\theta, \\
& \xi_{6}=\varphi .
\end{aligned}
$$

The instantaneous kinematic model (IKM) sets the derivative of its location as a function of the derivatives of the robotic arm configuration and of the location of the mobile platform:

$$
\dot{\boldsymbol{\xi}}=\frac{\partial \boldsymbol{f}}{\partial \boldsymbol{q}_{a}}\left(\boldsymbol{q}_{a}, \vartheta\right) \dot{\boldsymbol{q}}_{a}+\frac{\partial \boldsymbol{f}}{\partial \boldsymbol{\xi}_{p}}\left(\boldsymbol{q}_{a}, \vartheta\right) \dot{\boldsymbol{\xi}}_{p} .
$$

Remark that $\boldsymbol{f}$ is a function of $\boldsymbol{q}_{a}$ and $\boldsymbol{\xi}_{p}$, whereas its partial derivatives only depend on $\boldsymbol{q}_{a}$ and $\vartheta$. From equations (15) and (16):

$$
\frac{\partial \boldsymbol{f}}{\partial \boldsymbol{q}_{a}}\left(\boldsymbol{q}_{a}, \vartheta\right)=\left[\begin{array}{c}
J_{a_{1, \star}} C_{\vartheta}-J_{a_{2, \star}} S_{\vartheta} \\
J_{a_{1, \star}} S_{\vartheta}+J_{a_{2, \star}} C_{\vartheta} \\
J_{a_{3, \star}} \\
J_{a_{4, \star}} \\
J_{a_{5, \star}} \\
J_{a_{6, \star}}
\end{array}\right]
$$

$J_{a_{k, \star}}$ being the $k-t h$ line of the robotic arm Jacobian matrix and:

$$
\frac{\partial \boldsymbol{f}}{\partial \boldsymbol{\xi}_{p}}\left(\boldsymbol{q}_{a}, \vartheta\right)=\left[\begin{array}{ccc}
1 & 0 & -\left(\left(a+\xi_{a_{1}}\right) S_{\vartheta}+\left(b+\xi_{a_{2}}\right) C_{\vartheta}\right) \\
0 & 1 & \left(a+\xi_{a_{1}}\right) C_{\vartheta}-\left(b+\xi_{a_{2}}\right) S_{\vartheta} \\
0 & 0 & 0 \\
0 & 0 & 1 \\
0 & 0 & 0 \\
0 & 0 & 0
\end{array}\right] .
$$

We define the control of mobility of the mobile manipulator by $\boldsymbol{\eta}=\left[\begin{array}{ll}\boldsymbol{u}_{a}^{T} & \boldsymbol{\eta}_{p}^{T}\end{array}\right]^{T}$. Its dimension $\delta_{m}=n_{a}+\delta_{m_{p}}$ is termed as the degree of mobility of the mobile manipulator. The instantaneous location kinematic model (ILKM) sets the derivative of its location as a function of its control of mobility. According to (14) and (5), equation (17) writes:

$$
\dot{\boldsymbol{\xi}}=\frac{\partial \boldsymbol{f}}{\partial \boldsymbol{q}_{a}}\left(\boldsymbol{q}_{a}, \vartheta\right) \boldsymbol{u}_{a}+\frac{\partial \boldsymbol{f}}{\partial \boldsymbol{\xi}_{p}}\left(\boldsymbol{q}_{a}, \vartheta\right) R(\vartheta) \Sigma\left(\boldsymbol{\beta}_{s}\right) \boldsymbol{\eta}_{p} .
$$

So the ILKM writes:

$$
\dot{\boldsymbol{\xi}}=\bar{J}\left(\boldsymbol{q}_{a}, \vartheta, \boldsymbol{\beta}_{s}\right) \boldsymbol{\eta}
$$

with $\bar{J}\left(\boldsymbol{q}_{a}, \vartheta, \boldsymbol{\beta}_{s}\right)=\left[\frac{\partial \boldsymbol{f}}{\partial \boldsymbol{q}_{a}}\left(\boldsymbol{q}_{a}, \vartheta\right) \frac{\partial \boldsymbol{f}}{\partial \boldsymbol{\xi}_{p}}\left(\boldsymbol{q}_{a}, \vartheta\right) R(\vartheta) \Sigma\left(\boldsymbol{\beta}_{s}\right)\right] \mathrm{a}$ $m \times \delta_{m}$ matrix, which is not Jacobian.
We notice that the derivative of the mobile manipulator EE location does not depend on the derivative of the steering wheels orientation, for a given configuration, $i$. e . does not depend on the control of steerability $\boldsymbol{\zeta}_{p}$ of the platform.

We define the control of manoeuvrability of the mobile ma-

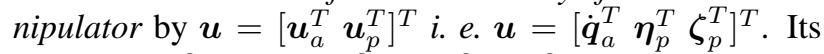
dimension, $\delta_{M}=n_{a}+\delta_{M_{p}}=\delta_{m}+\delta_{s_{p}}$ is termed as the degree of manoeuvrability of the mobile manipulator. If we define $\boldsymbol{z}=\left[\begin{array}{ll}\boldsymbol{\xi}^{T} & \boldsymbol{\beta}_{s}^{T}\end{array}\right]^{T}$, from (18) and (6), we get:

$$
\dot{\boldsymbol{z}}=B\left(\boldsymbol{q}_{a}, \vartheta, \boldsymbol{\beta}_{s}\right) \boldsymbol{u},
$$

with: $B\left(\boldsymbol{q}_{a}, \vartheta, \boldsymbol{\beta}_{s}\right)=\left[\begin{array}{cc}\bar{J}\left(\boldsymbol{q}_{a}, \vartheta, \boldsymbol{\beta}_{s}\right) & 0 \\ 0 & I_{N_{s}}\end{array}\right]$.

where $I_{k}, \forall k \in \mathbb{N}$, is the $k$-order identity matrix.

We also define the instantaneous configuration kinematic model (ICKM) by analogy with the case of the mobile platforms. When the platform ICKM is known, from equation (7) the mobile manipulator ICKM writes:

$$
\dot{\boldsymbol{q}}=S\left(\vartheta, \boldsymbol{\beta}_{s}, \boldsymbol{\beta}_{c}\right) \boldsymbol{u},
$$

where: $S\left(\vartheta, \boldsymbol{\beta}_{s}, \boldsymbol{\beta}_{c}\right)=\left[\begin{array}{cc}I_{n_{a}} & 0 \\ 0 & S_{p}\left(\vartheta, \boldsymbol{\beta}_{s}, \boldsymbol{\beta}_{c}\right)\end{array}\right]$.

\subsection{Example}

We consider the mobile manipulator shown in Figure 1, where $b=0$ for the sake of simplicity. We reduce the location to the planar position of the EE in the horizontal plane.

Arm modelling: The robotic arm is built from two $\left(n_{a}=\right.$ 2) bodies (of which lengths are respectively $a_{1}$ and $a_{2}$ ), articulated by two rotoïd joints with vertical parallel axes. $O_{0}$ is linked to the base of this robotic arm and $O_{3}$ is the center of the EE. The robotic arm configuration is given by the rotation angles $q_{a_{1}}$ and $q_{a_{2}}$ and so $\boldsymbol{q}_{a}=\left[\begin{array}{ll}q_{a_{1}} & q_{a_{2}}\end{array}\right]^{T}$. We define the EE location only by the planar position of $O_{3}$ in $\mathcal{R}_{0}: \boldsymbol{\xi}_{a}=\left[\xi_{a_{1}} \xi_{a_{2}}\right]^{T}\left(m_{a}=2\right)$. Therefore, the location space $\mathcal{M}_{a}$ is 2 - dimensional. The robotic arm KM is: $\xi_{a_{1}}=a_{1} C_{1}+a_{2} C_{12}, \xi_{a_{2}}=a_{1} S_{1}+a_{2} S_{12}$, where: $C_{i}=\cos q_{a i}, S_{i}=\sin q_{a i}, C_{i j}=\cos \left(q_{a i}+q_{a j}\right)$ and $S_{i j}=\sin \left(q_{a i}+q_{a j}\right), \forall i, j=1,2$. Thus, the IKM is $\dot{\boldsymbol{\xi}}_{a}=J_{a}\left(\boldsymbol{q}_{a}\right) \dot{\boldsymbol{q}}_{a}$, with:

$$
J_{a}\left(\boldsymbol{q}_{a}\right)=\left[\begin{array}{cc}
-\left(a_{1} S_{1}+a_{2} S_{12}\right) & -a_{2} S_{12} \\
a_{1} C_{1}+a_{2} C_{12} & a_{2} C_{12}
\end{array}\right] .
$$

Mobile manipulator modelling: The position $\mathrm{O}_{3}$ in the frame $\mathcal{R}$ is thus given by its Cartesian coordinates $\xi_{1}$ and $\xi_{2}$. The configuration is $\boldsymbol{q}=\left[q_{a_{1}} q_{a_{2}} \varphi_{1} \varphi_{2} \varphi_{3} x \text { y } \vartheta \beta_{3}\right]^{T}$. The control of manoeuvrability of the mobile manipulator is $\boldsymbol{u}=\left[\begin{array}{ll}\boldsymbol{u}_{a}^{T} & \boldsymbol{u}_{p}^{T}\end{array}\right]^{T}=\left[\begin{array}{lll}\dot{q}_{a_{1}} & \dot{q}_{a_{2}} & \eta_{p} \zeta_{p}\end{array}\right]^{T}$. The degree of mobility of the mobile manipulator is $\delta_{m}=3$ and its degree of manoeuvrability is $\delta_{M}=4$. According to the robotic arm KM and (15), the KM of this mobile manipulator is:

$$
\begin{aligned}
& \xi_{1}=x+\left(a+a_{1} C_{1}+a_{2} C_{12}\right) C_{\vartheta}-\left(a_{1} S_{1}+a_{2} S_{12}\right) S_{\vartheta}, \\
& \xi_{2}=y+\left(a+a_{1} C_{1}+a_{2} C_{12}\right) S_{\vartheta}+\left(a_{1} S_{1}+a_{2} S_{12}\right) C_{\vartheta} .
\end{aligned}
$$


From (12), (18) and the above KM, we get:

$$
\dot{\boldsymbol{\xi}}=\left[\begin{array}{c}
\dot{\xi}_{1} \\
\dot{\xi}_{2}
\end{array}\right]=\bar{J}\left(q_{a_{1}}, q_{a_{2}}, \vartheta, \beta_{3}\right)\left[\begin{array}{c}
\dot{q}_{a_{1}} \\
\dot{q}_{a_{2}} \\
\eta_{p}
\end{array}\right]
$$

where: $\bar{J}\left(q_{a_{1}}, q_{a_{2}}, \vartheta, \beta_{3}\right)=\left[\begin{array}{ccc}D_{2} & D_{1} & D_{4} \\ D_{6} & D_{5} & D_{7}\end{array}\right]$, with the following intermediate variables:

$$
\begin{aligned}
& C_{\vartheta 1}=\cos \left(\vartheta+q_{a_{1}}\right), S_{\vartheta 1}=\sin \left(\vartheta+q_{a_{1}}\right), \\
& C_{\vartheta 12}=\cos \left(\vartheta+q_{a_{1}}+q_{a_{2}}\right), S_{\vartheta 12}=\sin \left(\vartheta+q_{a_{1}}+q_{a_{2}}\right), \\
& D_{1}=-a_{2} S_{\vartheta 12}, D_{2}=-a_{1} S_{\vartheta 1}+D_{1}, \\
& D_{3}=-D S_{\beta_{3}}, D_{4}=C_{\vartheta} D_{3}+C_{\beta_{3}}\left(D_{2}-a S_{\vartheta}\right), \\
& D_{5}=a_{2} C_{\vartheta 12}, D_{6}=a_{1} C_{\vartheta 1}+D_{5}, \\
& D_{7}=S_{\vartheta} D_{3}+C_{\beta_{3}}\left(D_{6}+a C_{\vartheta}\right) .
\end{aligned}
$$

If we take into account (6) and (20), we also get:

$$
\dot{\boldsymbol{z}}=\left[\begin{array}{c}
\dot{\xi}_{1} \\
\dot{\xi}_{2} \\
\dot{\beta}_{3}
\end{array}\right]=B\left(q_{a_{1}}, q_{a_{2}}, \vartheta, \beta_{3}\right)\left[\begin{array}{c}
\dot{q}_{a_{1}} \\
\dot{q}_{a_{2}} \\
\eta_{p} \\
\zeta_{p}
\end{array}\right],
$$

with: $B\left(q_{a_{1}}, q_{a_{2}}, \vartheta, \beta_{3}\right)=\left[\begin{array}{cc}\bar{J}\left(q_{a_{1}}, q_{a_{2}}, \vartheta, \beta_{3}\right) & 0 \\ 0 & 1\end{array}\right]$.

The ICKM of the mobile manipulator:

$\left[\begin{array}{lllllll}\dot{q}_{a_{1}} & \dot{q}_{a_{2}} \dot{\varphi}_{1} \dot{\varphi}_{2} \dot{\varphi}_{3} \dot{x} \dot{y} \dot{\vartheta} \dot{\beta}_{3}\end{array}\right]^{T}=S\left(\vartheta, \beta_{3}\right)\left[\begin{array}{lll}\dot{q}_{a_{1}} & \dot{q}_{a_{2}} & \eta_{p} \zeta_{p}\end{array}\right]^{T}$ is immediately obtained using the platform ICKM (13):

$$
S\left(\vartheta, \beta_{3}\right)=\left[\begin{array}{cccc}
1 & 0 & 0 & 0 \\
0 & 1 & 0 & 0 \\
0 & 0 & \frac{1}{r}\left(D S_{\beta_{3}}-l C_{\beta_{3}}\right) & 0 \\
0 & 0 & -\frac{1}{r}\left(D S_{\beta_{3}}+l C_{\beta_{3}}\right) & 0 \\
0 & 0 & -\frac{D}{r} & 0 \\
0 & 0 & -D C_{\vartheta} S_{\beta_{3}} & 0 \\
0 & 0 & -D S_{\vartheta} S_{\beta_{3}} & 0 \\
0 & 0 & C_{\beta_{3}} & 0 \\
0 & 0 & 0 & 1
\end{array}\right] .
$$

\section{Discussion}

The usual notions of singularity, redundancy and manipulability must be discussed from this kinematic modelling. It can be remarked that mobility and steerability plays different roles with respect to the EE velocities. The class of mobile manipulators that have no controlled steering wheels leads to the simplest analysis : the usual techniques of arm kinematic control can be used by essentially replacing the map : $\dot{\boldsymbol{\xi}}_{a}=J\left(\boldsymbol{q}_{a}\right) \dot{\boldsymbol{q}}_{a}$ by the map $\dot{\boldsymbol{\xi}}=\bar{J} \boldsymbol{\eta}$, i. e. relying only on the ILKM.

When dealing with steering wheels, the properties are not so obvious to adapt. In a given configuration, the EE velocity $\dot{\boldsymbol{\xi}}$ depends only on the mobility control vector $\boldsymbol{\eta}$. Thus, singularities and manipulability still are defined by the ILKM. But, if redundancy means infinity of solutions for $\dot{\boldsymbol{q}}$ when $\dot{\boldsymbol{\xi}}$ is imposed at a given configuration $\boldsymbol{q}$, then the rank of $\bar{J}$ does not characterize redundancy. So, is the notion of redundancy of any use when there is no direct mapping between one generalized velocity component - the steering velocity - and the EE velocity ? or, is it necessary to adapt this notion to rely on an indirectly imposed law on steering wheels ?

Note also, that this modelling has proved to be useful to control the mobile manipulator when the EE motion is imposed [5] and that manipulability (relative to mobility control) has been used as a criterion to be maximized in a kinematic control scheme [6].

\section{References}

[1] J. C. Alexander and J. H. Maddocks. On the kinematics of wheeled mobile robot. International Journal of Robotic Research, 8(5):15-27, 1989.

[2] T. Arai. Robots with integrated locomotion and manipulation and their future. In IROS'96, pages 541-545, Osaka, Japan, November 1996.

[3] B. Bayle. Modélisation et commande cinématiques des manipulateurs mobiles à roues. $\mathrm{PhD}$ thesis, LAAS-CNRS, Toulouse University, 2001.

[4] B. Bayle, J.-Y. Fourquet, and M. Renaud. Génération des mouvements des manipulateurs mobiles : Etat de l'art et perspectives. Journal Européen des Systèmes Automatisés, 35(6):809-845, 2001.

[5] B. Bayle, J.-Y. Fourquet, and M. Renaud. Kinematic control of wheeled mobile manipulators. to appear in International Conference on Intelligent RObots and Systems (IROS), 2002.

[6] B. Bayle, J.-Y. Fourquet, and M. Renaud. Manipulability of wheeled mobile manipulators: Application to motion generation. submitted to the International Journal of Robotics Research, 2002.

[7] G. Campion, G. Bastin, and B. D'Andréa-Novel. Structural properties and classification of kinematic and dynamic models of wheeled mobile robots. IEEE Transactions on Robotics and Automation, 12(1):47-62, February 1996.

[8] W. Khalil and J. Kleinfinger. A new geometric notation for open and closed loop robots. ICRA'86, pages 75-79, 1986.

[9] P. Muir and C. Neuman. Kinematic modeling of wheeled mobile robots. Journal of Robotic Systems, 4(2):281-340, April 1987.

[10] J. Neimark and N. Fufaev. Dynamics of Nonholonomic Systems, volume 33. Translations of Mathematical Monographs, 1972.

[11] R. Paul. Robot Manipulators : Mathematics, Programming, and Control. MIT press, Cambridge, London, United Kingdom, 1981.

[12] T. Yoshikawa. Foundations of Robotics : Analysis and Control. The MIT Press, 1990. 